\title{
A TSD determination of a DL using two-point tactual stimuli applied to the back
}

\author{
WILLIAM N. BOYER, HENRY A. CROSS, GARY W. GUYOT, \\ and DONNELL M. WASHINGTON \\ Colorado State University, Fort Collins, Colo. 80521
}

A signal-detection (TSD) approach was used to determine a difference limen (DL) for supraliminal two-point tactual stimuli applied to the back. Using a standard stimulus of $80 \mathrm{~mm}$, the DL for four male Ss appeared to lie between $10-15 \mathrm{~mm}$. This DL was larger than one previously reported for the dorsal forearm, in harmony with the fact that the two-point threshold is larger for the back than for the forearm.

Recently, Cross, Boyer, \& Guyot (1970), following a suggestion by Underwood (1966), used a theory of signal-detection (TSD) approach in determining the amount of change necessary in a supraliminal two-point tactual stimulus for a $\mathrm{S}$ reliably to report the change. Such a procedure amounts to the determination of a DL and differs from the usual threshold measures one encounters in the literature on two-point thresholds. In the Cross et al (1970) study, the supraliminal stimuli were applied to the dorsal forearm. It was concluded that, despite some individual differences, an increase of $6 \mathrm{~mm}$ in the $47 . \mathrm{mm}$ standard was necessary to enable $S s$ to reliably report the change. This study employs the same methodology but with stimuli applied to the back. The question of concern was whether the larger two-point threshold reported for the back, $68 \mathrm{~mm}$ as against $40 \mathrm{~mm}$ (Hilgard, 1953), would produce a larger DL than that reported for the dorsal forearm (Cross et al, 1970). METHOD

The Ss were four male students enrolled at Colorado State University who were paid for their services. The apparatus consisted of four aesthesiometers, one being a standard and the other three being in 5-mm steps unidirectional above the standard. Since the middle of the back was chosen as the stimulus area, a standard length well above the two-point threshold of $68 \mathrm{~mm}$ was selected. The standard stimulus was $80 \mathrm{~mm}$ and the other three stimuli were 85,90 , and $95 \mathrm{~mm}$, respectively.

A TSD approach exactly like that

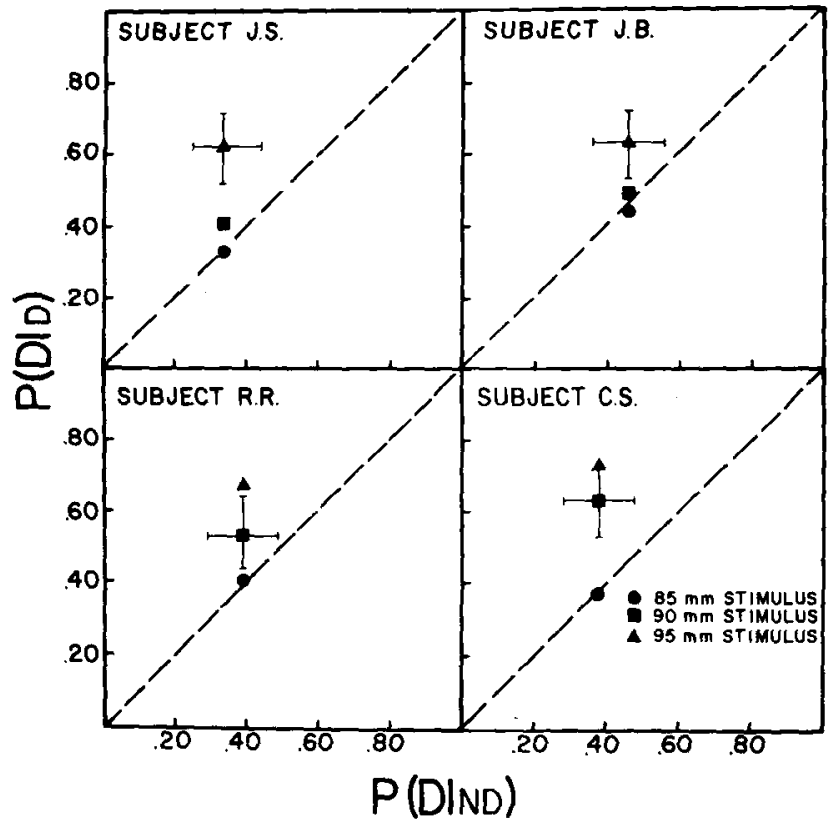

Fig. 1. $P(D / D)$ represents the proportion of "different" responses when the second stimulus was actually different from the standard, and $P(D / N D)$ represents the porportion of "different" responses when the test stimulus was not different from the standard. Bands for the $95 \%$ confidence intervals are shown for the various data points when there is a possible question about statistical significance. reported in the previous study was employed. The standard stimulus was always presented first in a two-stimulus sequence designated as a trial. The trials followed a random order in which one-half involved repetition of the standard, i.e., standard-standard, and one-half involved first the standard and then one of the three test stimuli.

Each $\mathrm{S}$ was told that his task was to make comparative judgments of a set of two stimuli presented successively. He was asked to respond "same" if the two stimuli of a trial were judged to be the same length and to respond "different" if they appeared to be different in length. If $S$ reported "different" when the second stimulus of a trial was indeed different, this constituted a "hit," but if he reported "different" during those trials in which the standard was repeated, this was scored as a "false alarm." In this way it was possible to obtain three ROC curves for each $\mathrm{S}$ which reflected his sensitivity under the test conditions of 85,90 , and $95 \mathrm{~mm}$. All other aspects of the procedure have been previously reported (Cross et al, 1970).

Each experimental session consisted of 96 trials, with a total of seven daily sessions. Thus, each standard-standard trial was repeated a total of 336 times, and each of the three standard-different combinations was presented a total of 112 times.

\section{RESULTS AND DISCUSSION}

To test statistically for sensitivity, the binomial data point in the unit square was calculated for each test stimulus and two 95\% confidence bands (one for the proportion of hits, the other for the proportion of false alarms) were calculated for each data point. These results for the four $\mathrm{Ss}$ are shown in Fig. 1.

It can be seen that for each $S$ all data points were ordered as predicted. For J.S. only the $95-\mathrm{mm}$ stimulus yielded significant sensitivity, and the $95-\mathrm{mm}$ data point was also different from both the 90 and the $85-\mathrm{mm}$ ones. S J.B. also did not display sensitivity until the $95-\mathrm{mm}$ test stimulus. S R.R. did, however, display significant sensitivity at both the 90 and 95-mm stimuli, and all three data points differed from each other. C.S. also displayed sensitivity at 90 and $95 \mathrm{~mm}$, but these latter points did not differ from each other.

Although there were again individual differences, the least difference from the standard that resulted in a reliable judgment of "different" was $10 \mathrm{~mm}$ for Ss R.R. and C.S. Ss J.S. and J.B. each required $15 \mathrm{~mm}$ to reliably report different. The DL, then, would seem to lie between 10 and $15 \mathrm{~mm}$. These results 
contrast with the study involving the dorsal forearm where the mean difference value, i.e., DL, for the four Ss tested was $6 \mathrm{~mm}$.

In summary, it has been shown that the DL for supraliminal two-point tactual stimuli applied to the back is greater than a comparable DL derived from tactual stimuli applied to the dorsal forearm. This is consistent with the fact that the two-point threshold is considerably greater for the back than for the dorsal forearm.
REFERENCES

CROSS, H. A., BOYER, W. N., \& GUYOT, G. W Determination of a DL using two-point tactual stimuli: A signal-detection approach. Psychonomic Science, 1970, 00, 000-000.

GREEN, D. M., \& SWETS, J. A. Signal detection theory and psychophysics. New York: Wiley 1966.

HILGARD, E. R. Introduction to psychology, New York: Harcourt, Brace, \& World, 1953.

UNDERWOOD, B. J. Experimental psychology. New York: Appleton-Century-Crofts, 1966.

\title{
Magnitude of model's apparent pain and ability to aid the model as determinants of observer reaction time*
}

\author{
ROBERT A. BARON $\dagger$ \\ University of South Carolina, Columbia, S.C. 29208
}

Thirty-two undergraduate females participated in an experiment designed to investigate the effects of magnitude of apparent suffering of a live peer model and the instructions concerning their ability to aid this individual on 0 reaction time (RT). It was predicted that under conditions where Os were informed that they could influence the suffering of the model by the speed of their responses, RT would decrease as the magnitude of pain evidenced by this individual increased. However, under conditions where Os were informed that they could not aid the model, it was expected that RT would actually increase as the degree of pain shown by this person increased. Results supported these predictions.

A number of recent experiments (e.g., Bandura \& Rosenthal, 1966; Berger, 1962; Craig \& Lowery, 1969) have demonstrated that exposure to the apparent pain and suffering of another person may elicit signs of physiological arousal among Os. An additional study (Di Lollo \& Berger, 1965) has reported that such experience may also produce significant decrements in $O$ reaction time (RT). Thus, it appears that apparent pain in another may influence both the physiological reactions and the overt behavior of Os.

A recent experiment (Baron, 1970) sought to extend the findings of the Di Lollo and Berger study by investigating the effects of three levels of apparent suffering on the part of a live peer model on O RT. In accordance with the findings reported by these authors and certain suggestions made by Berger (1962, p. 463),

*The author wishes to express his appreciation to Rollie O. Waters and Larry M. Cope for their assistance in the collection of the data, and to Murray $O$. Meetze for his aid in the construction of the experimental apparatus.

†Requests for reprints should be sent to the author at the Department of Psychology, University of South Carolina, Columbia, S.C. 29208.

it was hypothesized that $\mathrm{O}$ RT would decrease as the magnitude of pain cues emitted by the model increased. However, contrary to this prediction, it was found that $O$ RT actually increased significantly as the intensity of pain evidenced by the model increased.

The present study was designed to examine the possibility that the conflicting findings of the Di Lollo \& Berger (1965) and Baron (1970) experiments arose from certain procedural differences which led Os in the two studies to assume different degrees of control over the suffering of the model. In the Di Lollo and Berger study, conditions were arranged so that there was often a close temporal contiguity between $O s^{\prime}$ overt responses and the cessation of shocks to the model. As a result of this association, many Os may have assumed that they could influence the duration of the apparent shocks to the model. Thus, the decrements in RT that were observed may have been produced by their subsequent attempts to "aid" this individual.

In the Baron (1970) study, on the other hand, such a close temporal contiguity between $\mathrm{Os}^{\mathrm{s}}$ overt responses and cessation of shock to the model was lacking. Thus, it seems unlikely that Os in this study concluded that they could affect the suffering of this individual by varying the speed of their responses. Instead, they were faced with a situation in which they could observe the suffering of another individual but could not aid him in any manner. In order to reduce the aversiveness of this situation, they may have engaged in various self-generated competing responses, such as looking away from the model or focusing their attention on irrelevant stimuli in the experimental room. Such activities, which have been reported in previous studies (e.g., Bandura \& Rosenthal, 1966; Berger, 1962), may then have interfered with Os' RT by reducing their attention to the experimental task.

It was reasoned that if the above suggestions were accurate, instructions to Os regarding their ability to influence the shocks to the model would exert an important effect upon O RT. Specifically, it was predicted that under conditions where Os were informed that they could control the shocks to the model, O RT would decrease as the magnitude of pain cues emitted by the model increased. However, under conditions where Os were informed that they could not influence the shocks to the model, O RT would actually increase as the magnitude of pain cues emitted by this individual increased.

\section{OBSERVERS}

Thirty-two undergraduate females enrolled in sections of elementary psychology at the University of South Carolina participated in the experiment. Students took part in the study in order to fulfill a course requirement.

\section{APPARATUS}

The apparatus consisted of a Hunter timer (Model 111-C), a Lafayette digital stop clock (Model 5721), two stimulus panels, two telegraph keys, an electric buzzer, and one pair of finger electrodes. One stimulus panel contained red and white signal lights, while the second unit contained only a white light.

\section{DESIGN}

A 2 by 2 factorial design, based upon two levels of apparent shock to the model (low, high) and two levels of instructions regarding the ability of Os to influence the shocks to the model (independent, dependent), was employed. Eight Os were assigned randomly to each cell of this design.

\section{PROCEDURE}

The procedures of the present study were highly similar to those employed in a previous experiment (Baron, 1970). Thus, they will not be described in detail here. Briefly, Os observed a model (a confederate of the $E$ ) receive one of two levels of electric shock, and then responded 